\section{On the Use of Front-End Cascode Rectifiers Based on Normally-on SiC JFET and $\mathrm{Si}$ MOSFET}

\author{
Aitor Vazquez*, Alberto Rodriguez*, Marcos \\ Fernandez*, Marta M. Hernando*, Enrique Masset ${ }^{\dagger}$, \\ Javier Sebastian* \\ *University of Oviedo, Electronic Power Supply Systems \\ group
}

Edificio 3, Campus de Viesques s/n, 33204 Gijon, SPAIN

${ }^{\dagger}$ University of Valencia, Computer and Electronic Department,

Power Electronics Division University Burjassot, 24100, Valencia, SPAIN

\section{Corresponding author}

Aitor Vazquez

Edificio Departamental 3, Campus de Viesques $\mathrm{s} / \mathrm{n}$

33204, Gijón (SPAIN)

Phone: +34 985182578

Fax: +34985182138

Mail: vazquezaitor.uo@uniovi.es

Abstract.- The new wide band-gap semiconductor devices provide new properties to be explored. Normally-on Silicon Carbide (SiC) JFET power devices have several advantages, in particular low switching losses and the potential capabilities of high temperature and high reverse blocking voltage. Looking for improving the overall efficiency in power converters, new structures based on these power devices might be studied. In this paper, a cascode rectifier based on normally-on SiC JFET is presented and analyzed. This rectification structure can be applied as front-end rectifier stage for AC-DC power converters, increasing the overall efficiency of these topologies. A second cascode rectifier based on Silicon (Si) MOSFET is also studied, as a low cost alternative. A simple static forward characterization and a brief dynamic behavior analysis of the proposed cascode rectifier structure are made. Both cascode structures are compared with traditional $\mathrm{Si}$ rectifier diodes and front-end rectifiers, using an active PFC interleaved boost converter. As a result of this comparison, an efficiency improvement as high as two points is obtained. An additional OR gate based on a diode is also used as second test circuit to compare the proposed structures to the traditional Si rectifiers. A reduction between $75 \%$ and $55 \%$ of the total loss are obtained in this second experimental test.

Keywords: AC-DC converters, front-end rectifier, selfsynchronous rectifier (SSR), SiC JFET, Schottky diodes.
This paper has not been published or submitted previously. 


\section{On the Use of Front-End Cascode Rectifiers Based on Normally-on SiC JFET and Si MOSFET}

\section{INTRODUCTION}

AC-DC power supplies are one of the traditional fields in power electronics. Single-phase AC-DC converters are widely used in different applications, such as integrated lighting ballasts, telecommunication and computer equipment, battery chargers for mobile devices and also for electric and hybrid vehicles. Moreover, industrial AC and DC motor drives also use AC-DC power conversions. During the last decade, different solutions have been presented in order to improve these power supplies, regarding to achieve higher efficiency, lower volume and cost and higher reliability. In this sense, the use of the new wide band-gap semiconductors is one of the possible ways to overcome the AC-DC limitations. In particular, Silicon Carbide (SiC) devices exhibit a lot of advantages, well studied and presented in [1] and [2].

A higher reverse voltage capability, no reverse current and higher working temperature are the main potential advantages of this technology. In order to improve the overall efficiency in AC-DC converters, SiC devices have replaced $\mathrm{Si}$ semiconductors in this kind of power supplies. The first attempt to introduce $\mathrm{SiC}$ devices in AC-DC converters was replacing the ultra-fast diode with a high voltage SiC Schottky diode. Some good examples are studied in [3]-[5], where the SiC Schottky diode is used to avoid the reverse recovery current of the freewheeling diode, reducing the losses attached to this effect. A comparison between these new devices and $\mathrm{Si}$ ultra-fast diodes are also made in [6] and [7]. Due to the growing and development of $\mathrm{SiC}$ technology, other devices have been developed, not only high voltage Schottky diodes, but also transistors. Normally-on and normally-off $\mathrm{SiC}$ JFETs are ones of the most interesting devices and they are also commercially available [8, 9]. These new transistors have a high blocking voltage capability with very low on-resistance. On the other hand, the normally-on JFET should be driven with an inverter logic, different from the one used with normally-off switching devices. This means that the normally-on JFET is blocking when a negative voltage is applied to the gate (less than $-15 \mathrm{~V}$ ) and the device is conducting when $0 \mathrm{~V}$ (or a very low positive voltage) is applied to the gate. Comparison studies of these devices are made in [10] and [11], highlighting all the aforementioned advantages. Furthermore, some new structures based on these SiC semiconductor devices, just like cascodes based on normally-on JFETs, are studied in [12]-[15]. The main switching Si transistor can be replaced "pin-by-pin" by these new cascode structures; in [16] and [17] SiC JFET cascodes are proposed as main switching devices for
Power Factor Corrector (PFC) and boost converters, obtaining high efficiency.

The last element to be replaced in AC-DC converters is the front-end rectifier. Several techniques have been proposed to improve the rectification efficiency in single-phase rectifiers, such as bridgeless [18]-[20] or totem-pole circuits [21]. In this sense, some bidirectional rectifiers based on SiC JFETs are also studied in [22]. In [23]-[25] normally-on SiC JFET and Si MOSFETs are used as synchronous rectifiers (i.e. as controlled rectifier) with different driving circuits, in order to improve the overall rectifier efficiency. In the previous mentioned works, all the driving control circuitry is critical for generating the properly driving signals for the synchronous rectifier, especially when normally-on SiC JFETs are used. In order to avoid this problem, this paper presents two different cascode rectifiers to be applied as front-end rectifiers. The first one is a cascode rectifier based on a normally-on SiC JFET without any control circuitry, which can be seen as a self-driven synchronous rectifier (i.e. without any use of driving signal for the JFET). The second proposed structure replaces the high cost SiC JFET with a MOSFET, in order to achieve a low cost solution. This paper is organized as follows. In Section II both cascode rectifier structures are presented and analyzed. In Section III, a simple forward characterization of the cascode rectifier is made in order to highlight the advantages of this structure. Furthermore, a brief dynamic behavior comparison is done concerning to find the operating boundaries of the proposed cascode structure working at high frequency. An efficiency comparison between traditional $\mathrm{Si}$ rectifiers and these proposed structures are also made using a PFC interleaved boost converter. In addition, a second comparison is done with an OR gate based on a diode for parallel converters. All the experimental results obtained with this circuit are also summarized in Section III. Finally, some conclusions are outlined in Section IV.

\section{CASCODE RECTIFIER STRUCTURE}

\section{A) CASCODE RECTIFIER BASED ON SIC JFET}

The structure proposed as single rectifier is shown in Fig. 1. As can be seen, this structure is formed by a normally-on SiC JFET and a low voltage Schottky diode. The SiC JFET source is also connected to the cathode of the diode. Similarly, the SiC JFET gate is connected to the anode of the diode. This basic structure can be seen as a cascode using a Schottky diode instead of a MOSFET. This structure is similar for GaN rectifiers in [26]. The operation of this structure can be explained from Fig. 2 (a), where the voltage source is a sinusoidal one. 


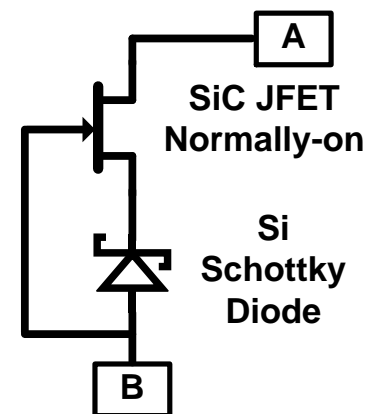

Fig. 1. Proposed cascode rectifier structure. SiC JFET and Schottky diode.

The equivalent circuits and the main waveforms are shown in Fig. 2 and in Fig. 3 (a) respectively. In the positive half cycle of the input voltage, the cascode rectifier is forward biased ( $V_{\text {cascode }}$ is positive). The $\mathrm{SiC}$ JFET could conduct (it is a normally-on device) but the Schottky diode does not conduct until the input voltage reaches its knee voltage $V_{\gamma-S H}$ (see Fig. 2 (c)). In other words, the ideal diode shown in Fig. 2 (b) is not conducting yet. When the input voltage is higher than $V_{\gamma-S H}$, then the Schottky diode is conducting (the ideal diode shown in the equivalent circuit is already conducting), see Fig. 2 (d). It should be noted that the SiC JFET is also conducting because its gate to source voltage is slightly positive (this is a normally-on device and, therefore, it conducts even with $0 \mathrm{~V}$ at the gate). As a consequence the complete device works as a diode whose knee voltage is just the knee voltage corresponding to the Si Schottky diode and whose dynamic resistance is the addition of the dynamic resistances of the Si Schottky diode and the SiC JFET. The voltage drop across this new device is lower than the voltage drop across a Si PIN diode for a wide range of conducting currents.

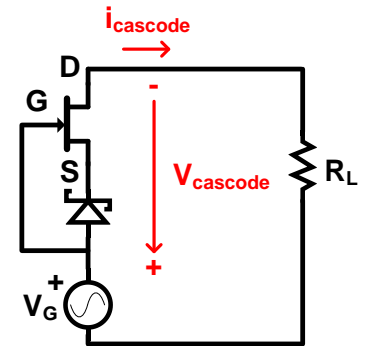

(a)

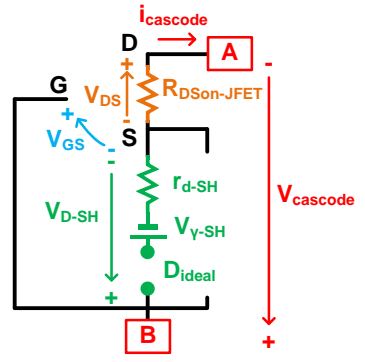

(c)

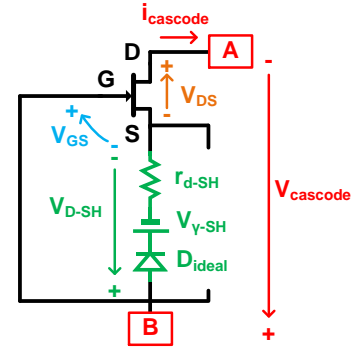

(b)

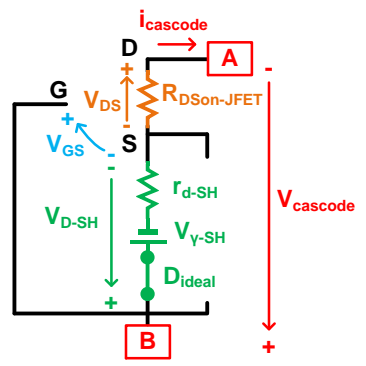

(d)

Fig. 2. Equivalent circuits for cascode rectifier based on SiC JFET when it is forward biased.

In the negative half cycle of the input voltage, the cascode rectifier is reverse biased ( $V_{\text {cascode }}$ is negative), as can be seen in Fig. 4. In this case, the Schottky diode is off (Fig. 4 (a)) because it is reverse biased. It should be noted that, at this moment, the whole cascode rectifier is not conducting, because the Schottky diode is blocking the reverse biased voltage. When the Schottky diode voltage, $V_{D-S H}$, reaches the pinch-off voltage of the SiC JFET (i.e. $V_{G S}=-15 \mathrm{~V}$ ) then the JFET will be turned-off and the SiC JFET will start blocking voltage, Fig. 4 (b). The equivalent waveforms for this case can be seen in Fig. 3 (b). It is important to remark that no control circuitry is needed to drive the SiC JFET.

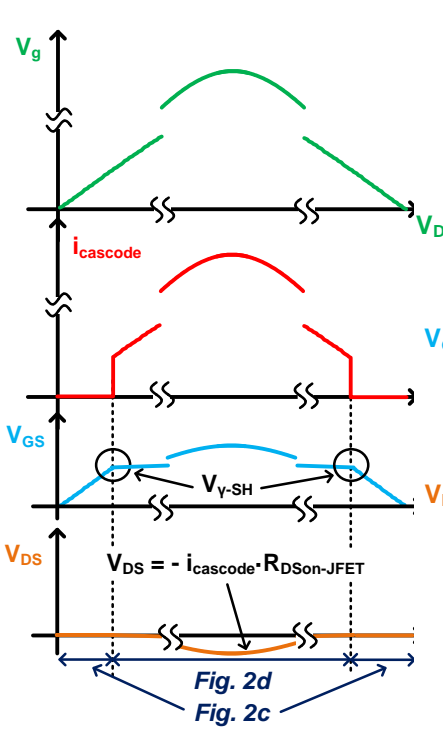

(a)

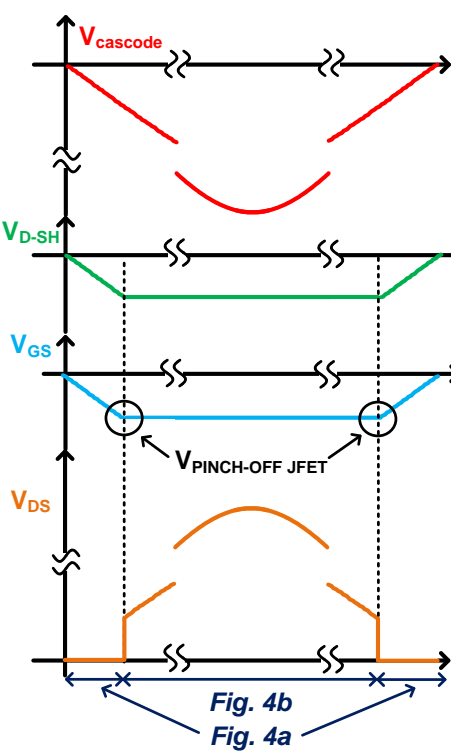

(b)
Fig. 3. Example waveforms for cascode rectifier based on $\mathrm{SiC}$ JFET. (a) When it is forward biased. (b) When is reverse biased.

The proposed cascode structure combines the high voltage blocking capability of the SiC JFET with the low knee voltage of the Si Schottky diode, thus obtaining a rectifier with excellent characteristics. As in the case of a standard rectifier, the proposed cascode rectifier does not need any control signal (for instance, none gate driver is needed for this device). The main disadvantage of the proposed cascode structure based on SiC JFET is the high cost of this element. Nowadays, these devices are commercially available but their price is higher than $\mathrm{Si}$ semiconductors. 


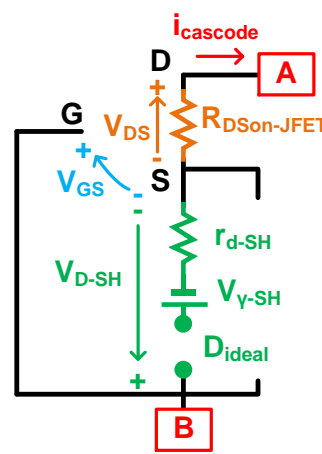

(a)

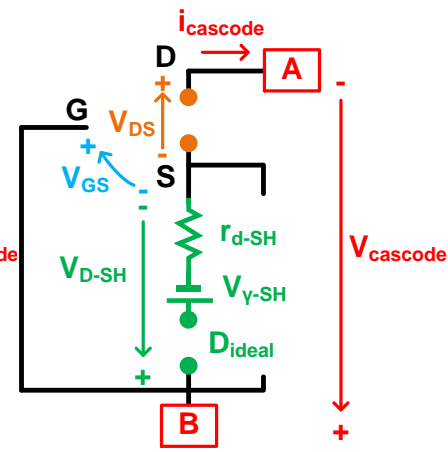

(b)

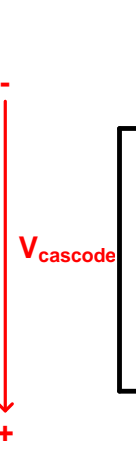

Fig. 4. Equivalent circuits for cascode rectifier based on $\mathrm{SiC}$ JFET when is reverse biased.

\section{B) CASCODE RECTIFIER BASED ON MOSFET}

In order to obtain a low cost but a high efficiency rectifier solution, a second structure is proposed (see Fig. 5 (a)). In this case, a Si MOSFET is used instead of a normally-on SiC JFET and a Zener diode is connected in parallel with the Schottky diode, to protect the MOSFET gate from overvoltage and spikes. Both diodes are connected in series with the MOSFET. In order to obtain low conduction losses, a forward DC voltage, $V_{B S T}$, is applied to the MOSFET gate. If this voltage is not applied, the MOSFET body diode could conduct, which will be a non-desirable situation due to the high conduction losses attached of this parasitic element. The $V_{G}$ voltage can be obtained from a simple bootstrap circuit, as shown in Fig. 5 (b). Capacitor $C_{B S T}$ is charged through the limiter resistance $R_{L I M}$. A voltage divider formed by $R_{A}$ and $R_{B}$ is used in order to keep the gate voltage under the limit of MOSFET breakdown specification.

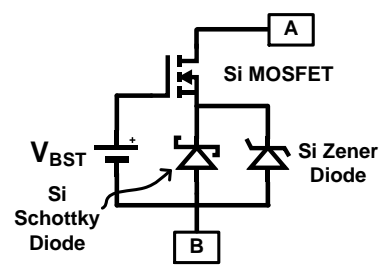

(a)

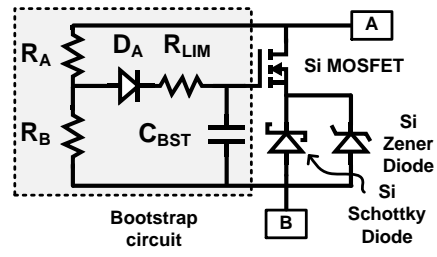

(b)
Fig. 5. Cascode rectifier based on Silicon MOSFET. (a) With an external voltage. (b) With a bootstrap circuit.

The behavior of this alternative cascode rectifier is identical to the aforementioned cascode rectifier based on $\mathrm{SiC}$ JFET. In fact, this structure can be seen just like a normally-on Si MOSFET which replaces the normally-on SiC JFET. The equivalent circuits and the main waveforms corresponding to the operation of this cascode rectifier are shown in Fig. 6, Fig. 7 and in Fig. 8. The only difference between the aforementioned circuits and figures and those corresponding to the cascode rectifier based on a SiC JFET is located just at the turn-off of the Si MOSFET. In the previous case, the SiC JFET turns-off just when the voltage across the Schottky diode reaches the pinch-off voltage of this JFET. On the other hand, the MOSFET turns-off when its gate to source voltage is lower than the threshold voltage $\left(\mathrm{V}_{\mathrm{GS}}<\mathrm{V}_{\mathrm{GS}}\right.$ threshold $)$. When the cascode rectifier is reverse biased, the gate to source voltage is the difference between the bootstrap voltage $\mathrm{V}_{\mathrm{BST}}$, and the reverse voltage across the Schottky diode, $\mathrm{V}_{\mathrm{D}-\mathrm{SHreverse}}=$ $-V_{D-S H}$; so $V_{G S}=V_{B S T}-V_{D-S H r e v e r s e}$. For low values of the voltage across the cascode rectifier, the MOSFET still has its channel and the reverse voltage is blocked by the Schottky diode. When the reverse voltage across the cascode rectifier $\left(\mathrm{V}_{\text {cascode }}\right)$ increases, the reverse voltage across the Schottky diode also increases $\left(\mathrm{V}_{\mathrm{D}-\mathrm{SHreverse}}\right)$. As

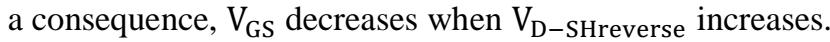
When the gate to source voltage decreases under threshold voltage then the MOSFET channel disappears and the main reverse voltage $\left(\mathrm{V}_{\text {cascode }}-\mathrm{V}_{\mathrm{D} \text {-SHreverse }}\right)$ is blocked by the MOSFET.

The Zener diode is used to protect the MOSFET gate against over-voltage. In this sense, this Zener diode prevents the MOSFET from damage due to negative gate to source voltage. To do that, its breakdown voltage must be lower than the bootstrap voltage plus the maximum value of the rated reverse gate to source voltage (typically $20 \mathrm{~V}$ ). It is important to remark that this Zener diode only would conduct when the cascode rectifier was reverse biased (as can be seen in Fig. 8 (c)). The extra losses added by this Zener are determined by the leakage currents of the MOSFET and Schottky diode, so, they are expected to be very low and their impact in the overall cascode rectifier efficiency can be neglected.

The cascode rectifier based on JFET does not need any driving circuitry, but the cascode rectifier based on MOSFET needs a bootstrap circuit instead. Nevertheless, this circuit is very simple (three resistors, a diode and a capacitor) and it can be adjusted easily to the characteristics of the final application varying only the voltage divider. It is important to remark that the cascode rectifier based on MOSFET loses the capability of withstanding very high voltage, due to the limit imposed by the Si MOSFET transistor. The maximum $V_{D S}$ in this kind of transistors is $800 \mathrm{~V}$, lower than the $1200 \mathrm{~V}$ for the $\mathrm{SiC}$ JFET used in the previous cascode rectifier. The onresistance of the MOSFET is also higher than the $\mathrm{SiC}$ JFET, so more conduction losses are expected in this cascode rectifier. Despite these two important disadvantages, the cascode rectifier based on MOSFET is still better, theoretically, than the Si diodes used as frontend rectifiers. In this sense, the behavior of the low voltage Schottky diode (with very low forward voltage and dynamic resistance) balances the previously mentioned drawbacks of this cascode rectifier. 


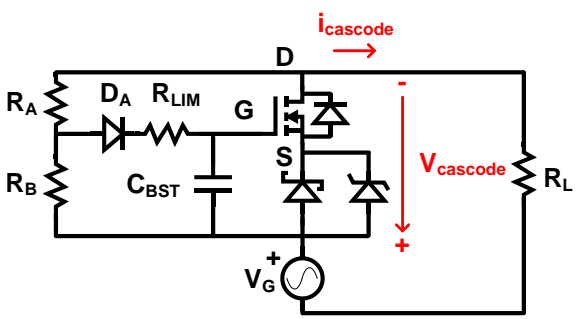

(a)

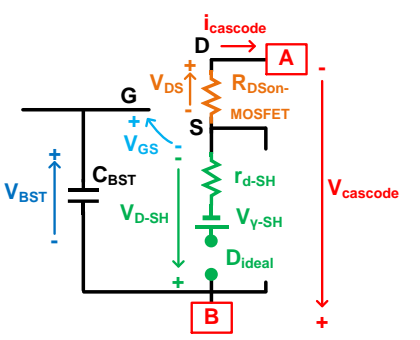

(b)

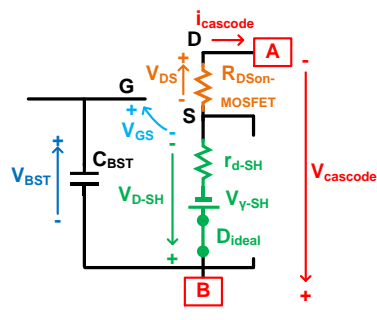

(c)
Fig. 6. Schematic behavior of cascode rectifier based on MOSFET when it is forward biased.

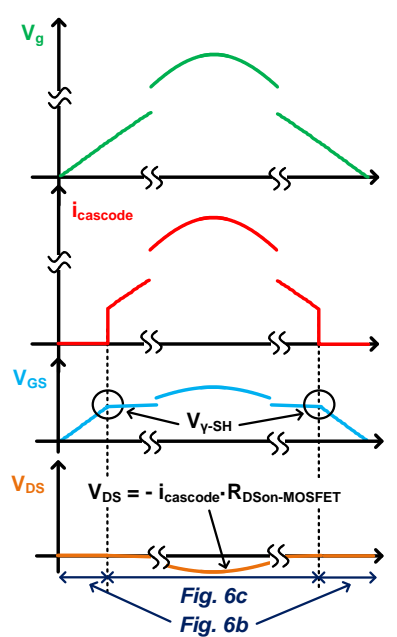

(a)

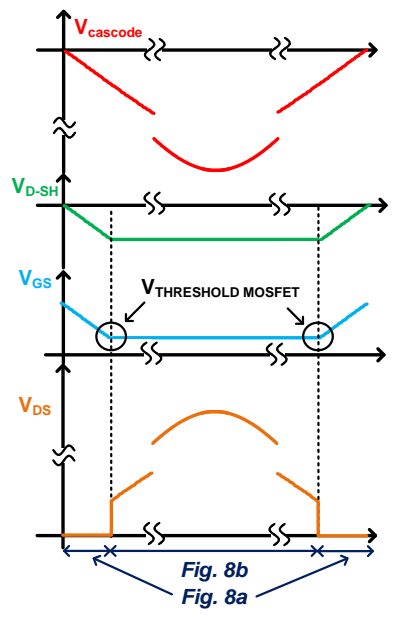

(b)
Fig. 7. Example waveforms for cascode rectifier based on MOSFET. (a) When it is forward biased. (b) When it is reverse biased.

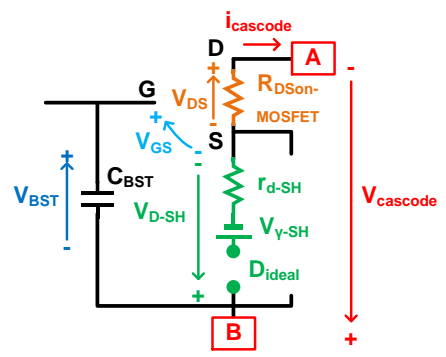

(a)

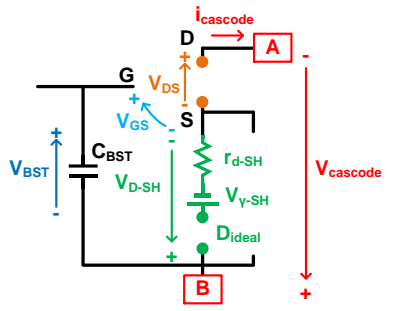

(b)

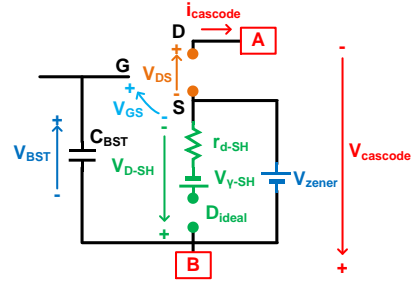

(c)
Fig. 8. Schematic behavior when the cascode rectifier based on MOSFET is reverse biased.

\section{EXPERIMENTAL RESULTS}

In this section, all the results obtained with different test circuits will be presented. First of all, a theoretical comparison of the proposed cascode rectifier and other Si rectifiers are made, in order to clarify the main advantages of this structure. The second part of this section will be dedicated to analyse the forward and reverse behaviour of the cascode rectifier with different circuits. Finally, the third part of this section will be employed to summarize the efficiency results obtained with three different circuits, in order to validate the theoretical advantages of both proposed cascode rectifier structures: a full-bridge front-end rectifier, a voltage doubler (passive PFC) and an interleaved boost converter (active PFC).

In all cases, the normally-on SiC JFET used is an SJDP120R085 from SemiSouth and the MOSFET is an IRFP15N60, both in a TO-247 package. Table summarizes the main characteristics of the SiC JFET and the MOSFET used in the proposed cascode rectifiers. In both cascode structures, the Schottky diode is the same, a $40 \mathrm{~L} 15 \mathrm{CW}$, in TO-247 package (15 V and $40 \mathrm{~A}$ ) from IR, and the Zener diode used in the cascode rectifier based on a MOSFET is also a $15 \mathrm{~V}$. In addition, several Si diodes have been used for testing and their characteristics are also shown in Table I.

Table I. SiC JFET and MOSFET used on each proposed cascode rectifiers.

\begin{tabular}{|c|c|c|c|c|}
\hline Device & $\begin{array}{c}\text { VDS } \\
{[\mathbf{V}]}\end{array}$ & $\begin{array}{c}\mathbf{I}_{\mathbf{D}} \\
{[\mathbf{A}]}\end{array}$ & $\begin{array}{c}\mathbf{R}_{\mathbf{D S}} \\
{[\mathbf{m} \mathbf{\Omega}]}\end{array}$ & Manufacturer \\
\hline $\begin{array}{c}\text { SJDP120R085 } \\
\text { (SiC JFET) }\end{array}$ & 1200 & 27 & 85 & SemiSouth \\
\hline $\begin{array}{c}\text { IRFP15N60 } \\
\text { (MOSFET) }\end{array}$ & 600 & 15 & 385 & Vishay \\
\hline
\end{tabular}

Table II. Different Silicon diodes used in the tested circuits.

\begin{tabular}{|c|c|c|c|}
\hline Diode & $\mathbf{V}_{\mathbf{R}}[\mathbf{V}]$ & $\mathbf{I}_{\mathbf{F}}[\mathbf{A}]$ & Manufacturer \\
\hline BR804 & 400 & 8 & EIC \\
\hline MUR4100 & 1000 & 4 & OnSemi \\
\hline GBU8J & 600 & 8 & Fairchild \\
\hline 20ETF08 & 800 & 20 & IR \\
\hline MUR3060PT & 600 & 30 & OnSemi \\
\hline GBPC1504 & 400 & 15 & Diodes INC \\
\hline
\end{tabular}




\section{A) A BRIEF THEORETICAL COMPARISON BETWEEN BOTH CASCODE RECTIFIERS AND SI DIODES}

In order to clarify the expected efficiency of both proposed cascode rectifiers, a brief theoretical analysis is presented in this section. To compare these rectifiers with the traditional $\mathrm{Si}$ rectifiers, this analysis is only focused on the conduction losses. Both cascode rectifiers behave like a Schottky diode connected with the equivalent onresistance of SiC JFET or MOSFET, when they are forward biased. This equivalent circuit can be used to obtain the rectifier static curves. Taking into account the JFET and MOSFET characteristics and using the datasheets of Si diodes listed in Table , the forward current against the forward voltage for each cascode rectifier and for each Si diode can be plotted. As can be seen in Fig. 9, both cascode rectifiers have lower voltage knee than the $\mathrm{Si}$ rectifiers. The slope of each rectifier is determined by the equivalent dynamic resistance of the whole rectifier. For instance, both cascode rectifiers have higher equivalent dynamic resistance than certain Si diodes. Due to this, the cascode rectifier only has lower static losses than the $\mathrm{Si}$ diode up to a maximum forward current value (at a maximum forward voltage). This value is determined by the cross of both static curves. Hence, these maximum forward current values are highlighted in red circles for cascode rectifier based on a SiC JFET, and they are remarked in blue circles for cascode rectifier based on a MOSFET. Above this forward current, it is expected that both cascode rectifiers have higher losses than the $\mathrm{Si}$ diodes. Nevertheless, these maximum current values are quite high for the cascode rectifier based on a SiC JFET, so this proposed solution is still interesting.

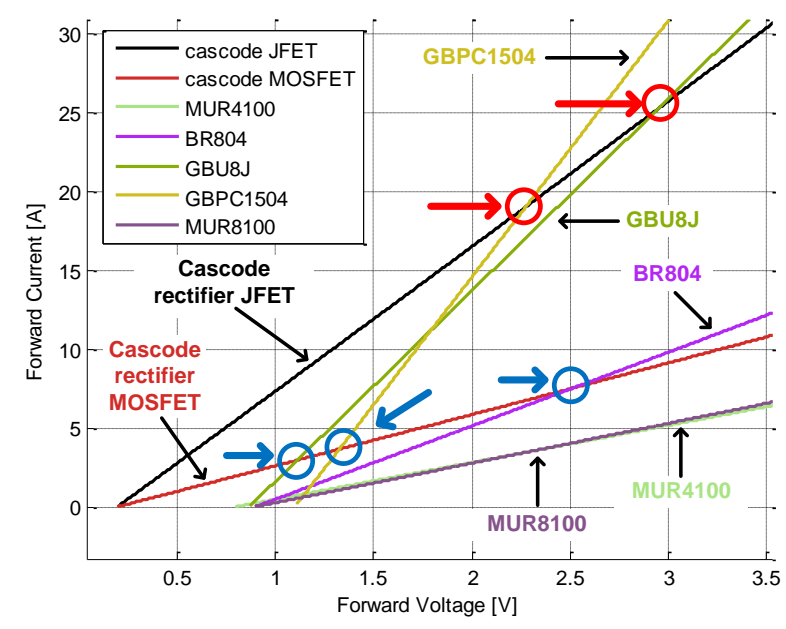

Fig. 9. Forward current against forward voltage for both cascode rectifiers and the Si diodes listed in Table II.

\section{B) FORWARD CHARACTERIZATION OF THE CASCODE RECTIFIERS}

In order to obtain a brief characterization of the proposed cascode rectifier structure and to validate the previously mentioned theoretical behavior, simple forward measurements are made. For instance, the circuit shown in Fig. 10 is used to measure the forward current and forward voltage of the cascode rectifier.

For this characterization, the cascode rectifier structure is compare with two different full bridge rectifiers GBPC1504 and GBU8K (equivalent to GBU8J) listed in Table II. All comparisons have been established using a single diode of each full bridge rectifier. The theoretical and measured forward currents are shown in Fig. 11. As can be seen in Fig. 11 (a), the best theoretical behavior is expected for synchronous rectification (green plot), which has not any knee voltage, but it needs an extra control circuitry and logic. The red line plotted in Fig. 11 is the forward current expected for cascode rectifier. This proposed structure has a lower knee voltage than the one corresponding to a single diode of the $\mathrm{Si}$ full bridge rectifiers (black and blue lines) but it also has a lower slope due to the additional on-resistance of the JFET (i.e. the equivalent dynamic resistance of the whole cascode rectifier is the addition of the dynamic resistance of the Schottky diode and the on-resistance of the JFET). The measured forward current for the cascode rectifier and for both full bridge rectifiers are shown in Fig. 11 (b). As can be seen, the cascode rectifier has a knee voltage close to $0.2 \mathrm{~V}$ (like the knee voltage of the low voltage Si Schottky diode used in the cascode), but it has a higher dynamic resistance (the slope of the red line is lower than the slope of the full bridge rectifiers, the black and blue lines). Therefore, the behavior of the cascode rectifier is like a low voltage Si Schottky diode when it is forward biased as expected to be.

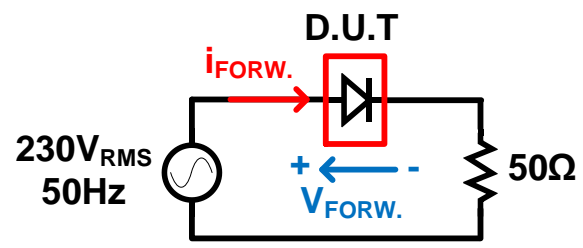

Fig. 10. Test circuit used for a simple forward characterization. 


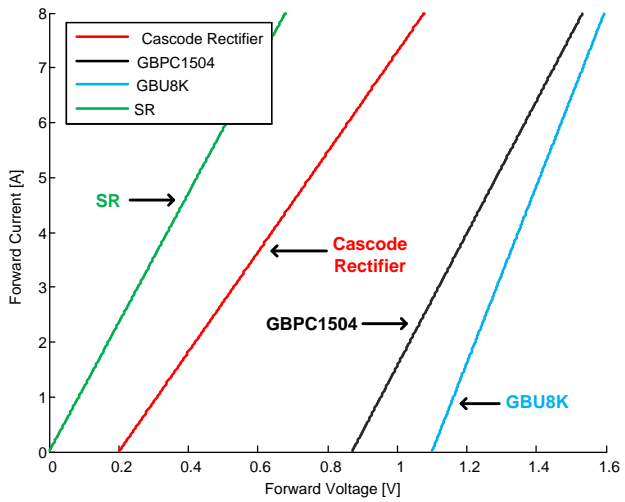

(a)

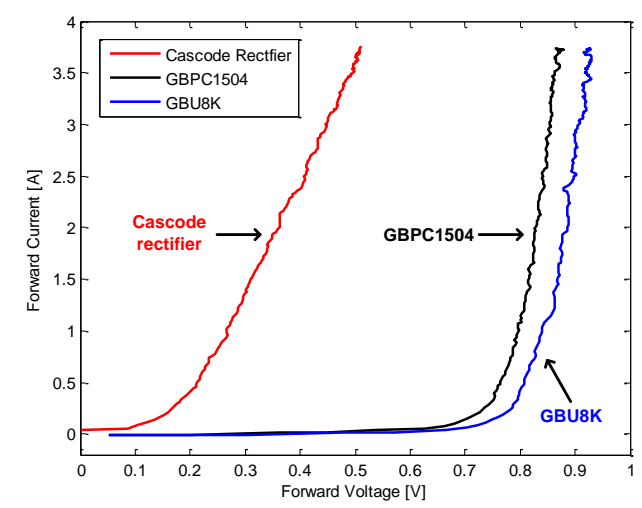

(b)

Fig. 11. Forward current versus forward voltage. (a) Theoretical. (b) Measured.

\section{C) DYNAMIC BEHAVIOR COMPARISON OF THE CASCODE RECTIFIERS}

Due to its better forward performance, the cascode rectifier can obtain better results than an equivalent high voltage PIN Si diode, working at low frequency. However, the dynamic behavior of the proposed cascode rectifier is not studied yet. Therefore, in this section a very simple dynamic behavior comparison is made between the cascode rectifier based on $\mathrm{SiC}$ JFET and two single rectifier diodes. A single phase DC-DC boost converter is used as test circuit, in order to obtain the behavior of the diode under test when it is working at high frequency. The main specifications of the boost converter used as test circuit for the cascode rectifier based on SiC JFET are shown in Fig. 12. Two different conduction modes are proposed as test benches: the boost converter working in Discontinuous Conduction Mode (DCM, see Fig. 12 (a)) or in Continuous Conduction Mode (CCM, see Fig. 12 (b)). In DCM, the $d i / d t$ ratio is $1.5 \mathrm{~A} / \mu$ s whilst in CCM this ratio is $0.1 \mathrm{~A} / \mu \mathrm{s}$. These different ratios are obtained by modifying the inductance value and the output voltage. In both cases the switching frequency is $100 \mathrm{kHz}$.

The operating waveforms of the boost converter in both conduction modes are shown in Fig. 13. It is important to remark that DCM operating mode is adjusted to achieve soft-switching condition in the transistor (i.e. Zero Voltage Switching). The inductance value and duty cycle of this conduction mode are selected very close to the values of Boundary Conduction Mode (BCM). On the other hand, in CCM both devices are working in hardswitching condition (i.e. the MOSFET is turning-on at no zero voltage and the diode is turning-off at no zero current).

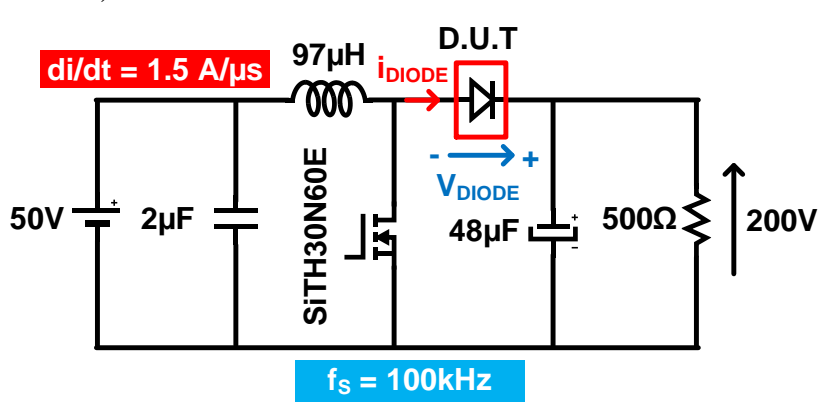

(a)

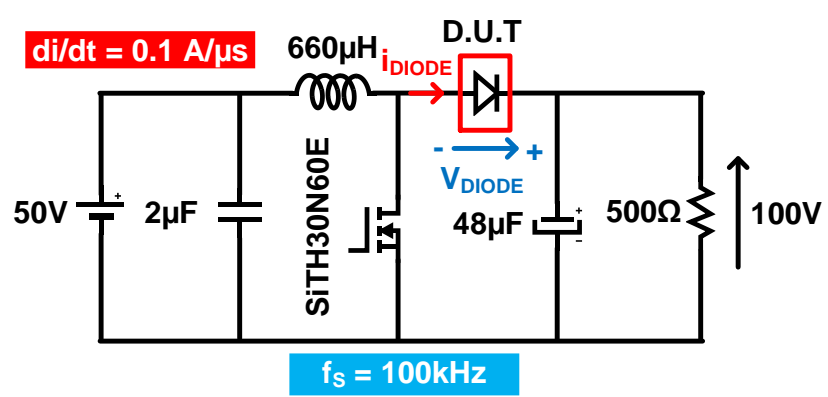

(b)

Fig. 12. Test circuits for reverse characterization. (a) Discontinuous Conduction Mode. (b) Continuous Conduction Mode.

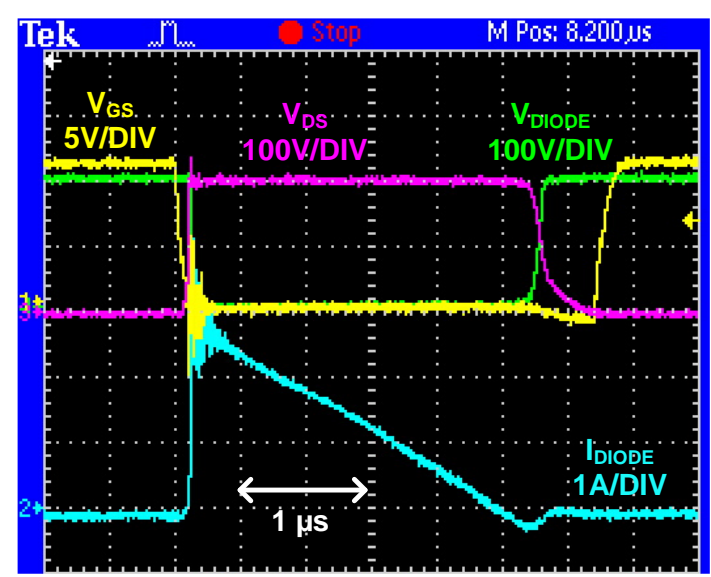

(a) 


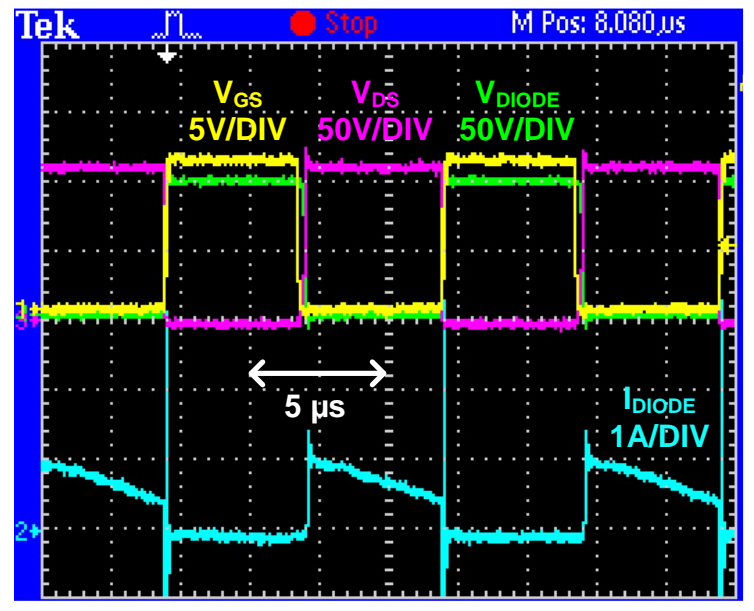

(b)

Fig. 13. Example operating waveforms of the boost converter. (a) Working in DCM. (b) Working in CCM.

Taking into account the previously mentioned conditions and specifications, different measurements are made for the proposed cascode rectifier and for Si diodes MUR30360PT and MUR4100 (the main characteristics of these devices are summarized in Table ). A SDT1260C $\mathrm{SiC}$ diode from Infineon is also used in the comparison, in order to establish the best possible performance, regarding the reverse recovery process. The main characteristics of this diode are $V_{R}=600 \mathrm{~V}$ and $I_{F}=12 \mathrm{~A}$.

The reverse recovery process for the different diodes is shown in Fig. 14. As can be seen, the lowest reverse recovery current is obtained for the $\mathrm{SiC}$ diode. The proposed cascode rectifier based on SiC JFET behaves slightly worse than a high voltage ultra-fast diode, regarding the maximum reverse peak value and the reverse recovery time.

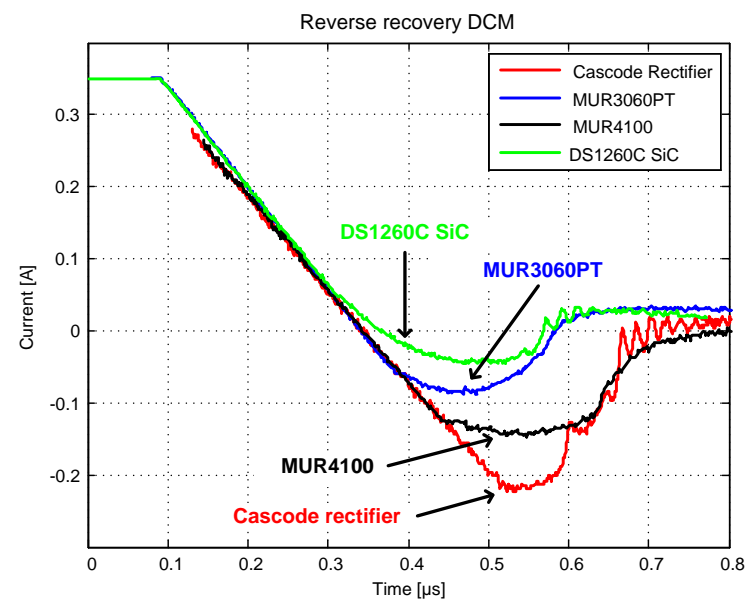

Fig. 14. Reverse recovery behavior.
In spite of the fact that the reverse recovery process is not as fast as in an ultra-fast Si diode, it is important to take into account that the proposed cascode rectifier structure can withstand a high reverse voltage (i.e. $1200 \mathrm{~V}$, twice than the other diodes used in this comparison), even with the same forward current.

\section{D) EXPERIMENTAL RESULTS IN A PFC INTERLEAVED BOOST CONVERTER}

In order to test the aforementioned advantages of the proposed structures, these cascode rectifiers are used as front-end rectifier in a PFC interleaved boost converter. The schematic for the two proposed cascode rectifier structures as front-end rectifiers can be seen in Fig. 15 (a) and Fig. Fig. 15 (b). For the cascode rectifier based on MOSFET, the bootstrap circuit is set up with $R_{A}=$ $3.9 \mathrm{M} \Omega, R_{A}=150 \mathrm{k} \Omega, R_{L I M}=150 \mathrm{k} \Omega, C_{B S T}=10 \mu \mathrm{F}$ and $\mathrm{D}$ is a $1 \mathrm{~N} 4148 \mathrm{PN}$ diode. A picture of the two cascode rectifier prototypes is shown in Fig. 16.

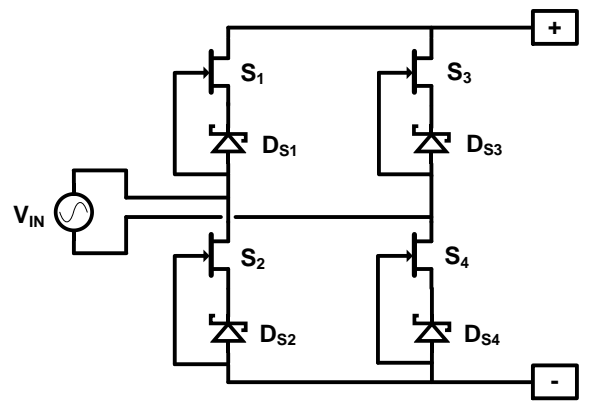

(a)

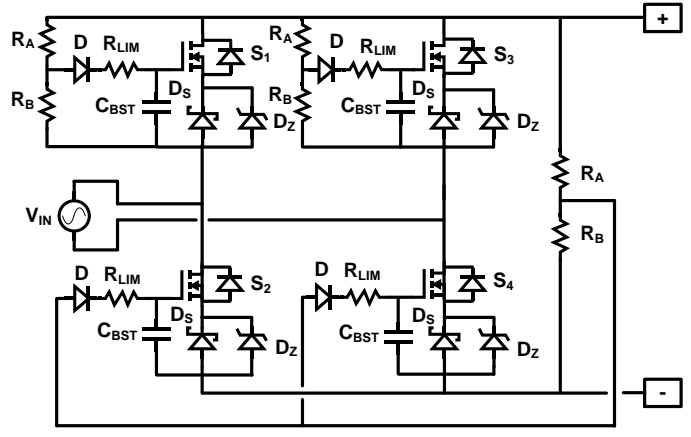

(b)

Fig. 15. Full bridge rectifier. (a) With cascode rectifiers based on SiC JFET. (b) With cascode rectifiers based on MOSFET.

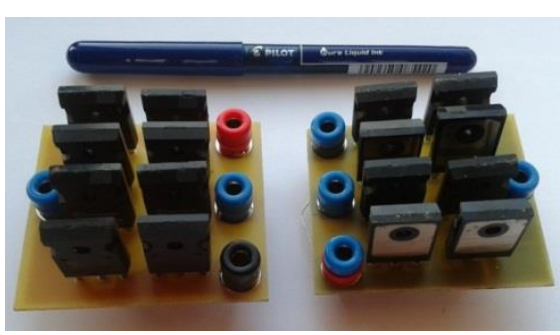

Fig. 16. Picture of the full bridge cascode rectifier. Left, cascode rectifier based on SiC JFET. Right, cascode rectifier based on MOSFET. 
The test circuit is an AC-DC PFC interleaved boost converter. The input voltage in this case is $230 \mathrm{~V}_{\mathrm{RMS}}$, $50 \mathrm{~Hz}$. The output voltage is $550 \mathrm{~V}$. The output power is varying from 200 to $1200 \mathrm{~W}$. This converter is working in closed loop with an average current mode multiplier control based on the integrated circuit UCC28070 from Texas Instruments.

Both cascode rectifiers are compared with a GBPC1508 full-bridge rectifier based on Si diodes. The inductor, output capacitor, freewheeling diode and main transistor used are summarized in Fig. 17. Experimental results for this boost converter are shown in Fig. 18. As can be seen, an improvement of efficiency of 1.2 points is obtained with cascode rectifier based on SiC JFET at full load. This improvement is about 0.8 points for cascode rectifier based on MOSFET. At half load, the efficiency of the cascode rectifier based on SiC JFET is 3 points higher than the Si full-bridge. An improvement of efficiency of 2.2 points is achieved for the cascode rectifier based on MOSFET at this power. At light load, there are 2 points between the efficiency of both cascode rectifiers and the $\mathrm{Si}$ full-bridge one. In all cases, the input power factor is fairly the same (0.999 at full load) and no distortion in the converter waveforms is observed.

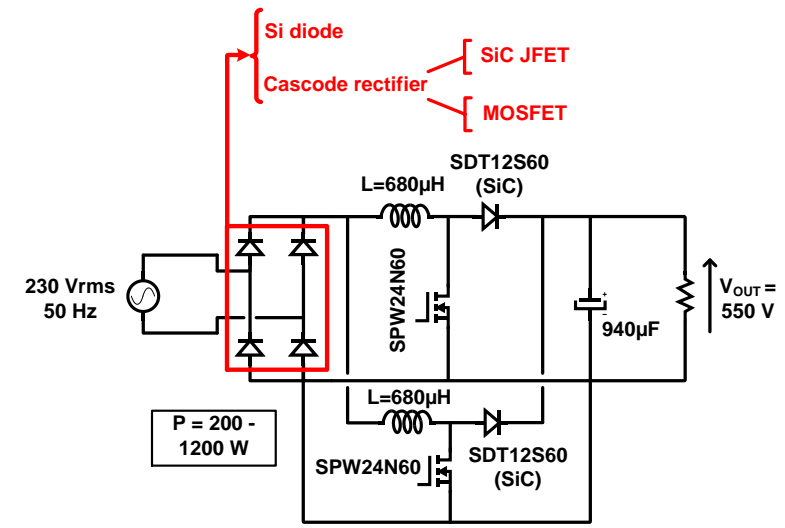

Fig. 17. Test circuit: two phase interleaved PFC boost converter.

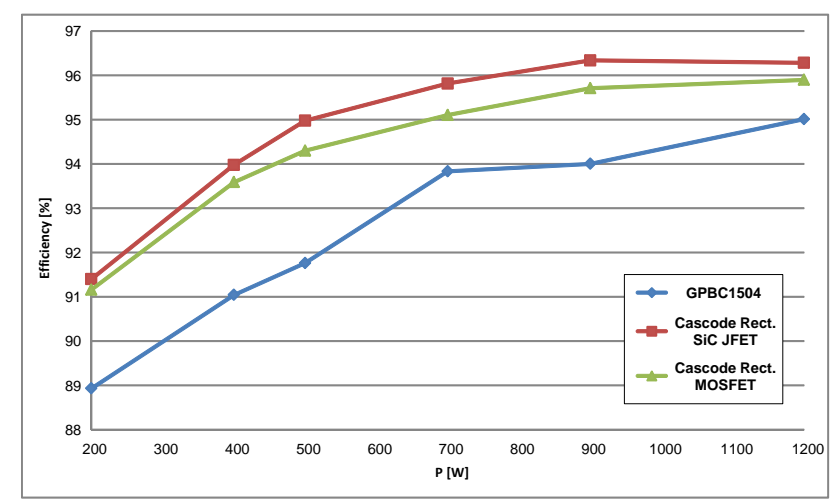

Fig. 18. Measured efficiency for PFC interleaved boost converter.

\section{E) EXPERIMENTAL RESULTS FOR AN OR GATE BASED ON DIODE}

In this case, a simple OR gate circuit is used to validate the advantages of the proposed cascode rectifier structures. The OR gate structure based on a diode is widely used when two or more converters are parallelized together to supply a load. A simple example of this can be found in Fig. 19. The two converters, A and B, are working together, but converter $\mathrm{A}$ is supplying an output voltage higher than converter B. So, only one converter (A) provides the whole power to the load, whilst the other one is off (converter B), because the OR diode is reverse biased. If converter A fails, then its output voltage decreases drastically. In this case, the OR diode will be turned-on and converter B will start to supply the power to the load. Converter A will be disconnected, because the OR gate diode is reverse biased.

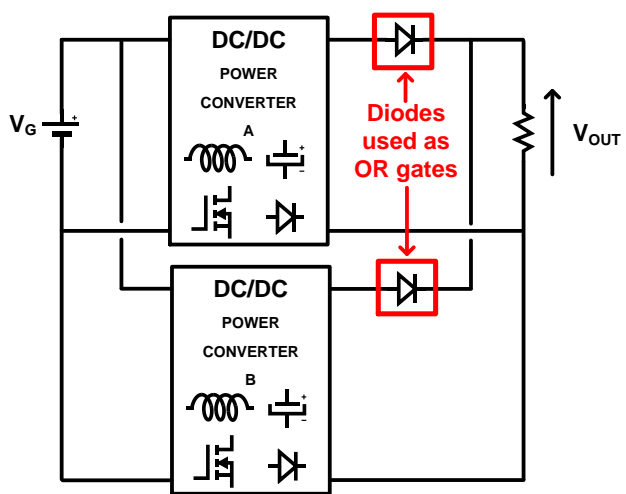

Fig. 19. Simple example of OR gates based on diodes for two parallel DC/DC converters.

This simple circuit is widely used in aerospace and automotive applications, where the reliability is the main objective and two or more power converters are used in parallel as a redundant power supply. Even more, this technique is also applied in low cost power supplies, because no high level control (i.e. a microcontroller) is needed to manage both converters in case of fail.

In an OR gate based on diodes, the conduction loss of the diode determines the overall efficiency of the whole power supply. Thus, this is an excellent scenario to test the proposed cascode rectifier structure. Two different Si diodes (MUR4100 and MUR840) are compared with the cascode rectifier based on a SiC JFET working as an OR gate. The output voltage is $300 \mathrm{~V}$ and the total diode loss is measured at two different output power, $300 \mathrm{~W}$ and 600 W. The experimental results are summarized in Table III and Fig. 20.

Table III. Total loss measured for each device used in OR gate circuit.

\begin{tabular}{|c|c|c|}
\hline Device & $\begin{array}{c}\text { Loss [W] @ } \\
\mathbf{P = 3 0 0 W}\end{array}$ & $\begin{array}{c}\text { Loss [W] @ } \\
\mathbf{P = 6 0 0 W}\end{array}$ \\
\hline MUR4100 & 1.05 & 2.12 \\
\hline MUR840 & 0.76 & 1.60 \\
\hline $\begin{array}{c}\text { Cascode rectifier } \\
\text { SiC JFET }\end{array}$ & 0.26 & 0.714 \\
\hline
\end{tabular}




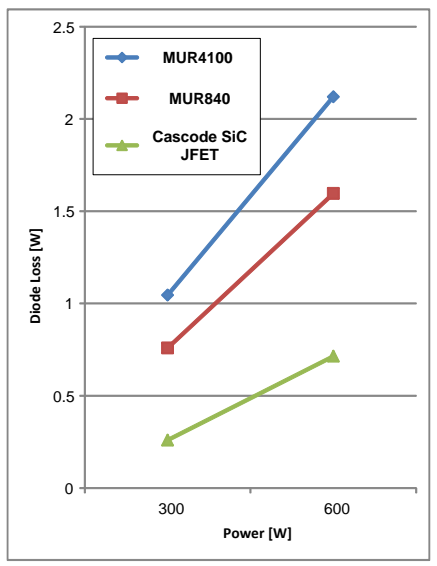

Fig. 20. Total diode loss measured for the OR gate circuit.

As can be seen, the cascode rectifier based on a SiC JFET has lower total loss than the MUR840 and MUR4100, as it is expected. The total loss reduction of the proposed cascode rectifier varies between $75 \%$ and $55 \%$ at each output power and for each $\mathrm{Si}$ diode. These measurements confirm the excellent conduction properties of the proposed cascode rectifiers.

\section{CONCLUSIONS}

AC-DC converters are widely used nowadays in many different applications. In the last years, new techniques to improve the overall efficiency of these converters have been investigated. New wide band-gap semiconductors, such as $\mathrm{SiC}$ ones, offer the opportunity to explore new ways to overcome the boundaries of efficiency in AC-DC converters. In particular, normally-on SiC JFET is one of the most interesting devices, due to the high blocking voltage capability, very low on-resistance and high potential temperature working range.

Focused in this kind of technology, two different cascode rectifier structures are presented and studied in this paper, looking for improving the efficiency of the front-end rectifiers used in AC-DC converters. The proposed cascode rectifier based on normally-on $\mathrm{SiC}$ JFET, combines the low losses of a low voltage drop of a Si Schottky diode in forward bias with the capability to withstand high voltage of a SiC JFET, when the cascode rectifier is reverse biased. It should be note that no control circuitry is needed in this cascode structure. However, the high cost of the SiC JFET is the main disadvantage of this solution. In order to solve or minimize this limitation, a second cascode rectifier based on a MOSFET is also studied. In this case, a bootstrap circuit is needed, due to the use of the MOSFET as a normally-on device. Nevertheless, the additional bootstrapping circuit needs a few low-power elements (three resistors, two diodes and a capacitor) and it can be adjusted easily depending on the final application where the rectifier will be used. In this sense, both cascode rectifiers could be used as high efficient self-driven synchronous rectifiers and they could be built in a small integrated component. Even more, an integrated version of a cascode rectifier could be developed, just implementing a high voltage normally-on Si JFET (with a very low on-resistance) and a Si Schottky diode (with a very low forward voltage drop) together, obtaining, as a result, a device with excellent conduction and blocking properties, reducing costs and parasitic effects.

With a simple forward characterization, the aforementioned theoretical advantages of the proposed cascode rectifier are confirmed. On the other hand, as a result of a brief dynamic behavior comparison, the cascode rectifier based on $\mathrm{SiC}$ JFET behaves slightly worse than a traditional $\mathrm{Si}$ ultra-fast diode, in terms of reverse recovery current and reverse recovery time. Thus, the proposed cascode rectifier could be used as front-end rectifier (i.e. when it has to work at low frequency, where the advantages of the forward behavior are more significant than the disadvantages of the reverse recovery performance), to improve the efficiency of these particular rectifiers. Adopting these structures, the efficiency of an AC/DC converter could be improved by direct droping replacement of a $\mathrm{Si}$ front-end rectifier, without modifying the original topology (as it occurs in the bridgeless techniques) or adding any extra control circuitry (as it happens in the synchronous rectification approaches).

A PFC interleaved boost converter has been built and measured in the laboratory, in order to test these cascode rectifiers and to compare them with traditional $\mathrm{Si}$ front-end rectifiers. The results obtained with both cascode rectifiers have an efficiency 3 points higher at light load and up to 2 points higher at full load than the traditional $\mathrm{Si}$ full bridge rectifier, operating in the same conditions.

A second test circuit, based on an OR gate for parallel converters, is also used to confirm the advantages of the proposed cascode rectifiers from the conduction point of view. A reduction between $75 \%$ and $55 \%$ is measured for the cascode rectifier compare to traditional $\mathrm{Si}$ rectifiers.

As a consequence of this study, the proposed cascode rectifiers can be used as front-end rectifiers or as low frequency rectifiers to improve the overall efficiency in those applications where the conduction losses have to be minimized.

\section{ACKNOWLEDGEMENTS}

This work is supported by the Spanish Government under projects MICINN-10-DPI2010-21110C0201 and CONSOLIDER MICINN-10-CSD2009-00046, FPU student grant AP2008-03380 and FPI grant BES2011-044114.

\section{REFERENCES}

[1] J. Biela, M. Schweizer, S. Waffler and J. Kolar, "SiC versus $\mathrm{Si}$-Evaluation of Potentials for Performance Improvement of Inverter and DC-DC Converter Systems by SiC Power Semiconductors," 
Industrial Electronics, IEEE Transactions on, vol. 58, no. 7, pp. 2872-2882, Jul. 2011.

[2] D. Jiang, R. Burgos, F. Wang and D. Boroyevich, "Temperature-Dependent Characteristics of $\mathrm{SiC}$ Devices: Performance Evaluation and Loss Calculation," Power Electronics, IEEE Transactions on, vol. 27, no. 2, pp. 1013-1024, Feb. 2012.

[3] G. Spiazzi, S. Buso, M. Citron, M. Corradin and R. Pierobon, "Performance evaluation of a Schottky $\mathrm{SiC}$ power diode in a boost PFC application," Power Electronics, IEEE Transactions on, vol. 18, no. 6, pp. 1249-1253, Nov. 2003.

[4] B. Lu, W. Dong, Q. Zhao and F. Lee, "Performance evaluation of CoolMOS and $\mathrm{SiC}$ diode for singlephase power factor correction applications," Applied Power Electronics Conference and Exposition, 2003. APEC '03. Eighteenth Annual IEEE, vol. 2.

[5] F. Chimento, S. Musumeci, A. Raciti, M. Melito and G. Sorrentino, "Super-Junction MOSFET and $\mathrm{SiC}$ Diode Application for the Efficiency Improvement in a Boost PFC Converter," IEEE Industrial Electronics, IECON 2006 - 32nd Annual Conference on.

[6] M. Hernando, A. Fernandez, J. Garcia, D. Lamar and $\mathrm{M}$. Rascon, "Comparing $\mathrm{Si}$ and $\mathrm{SiC}$ diode performance in commercial AC-to-DC rectifiers with power-factor correction," Industrial Electronics, IEEE Transactions on, vol. 53, no. 2, pp. 705- 707, Apr. 2006.

[7] C. N.-M. Ho, H. Breuninger, S. Pettersson, G. Escobar and F. Canales, "A Comparative Performance Study of an Interleaved Boost Converter Using Commercial Si and SiC Diodes for PV Applications," Power Electronics, IEEE Transactions on, vol. 28, no. 1, pp. 289-299, Jan. 2013.

[8] SemiSouth, "http://semisouth.com/," [Online]. [Accessed 1211 2012].

[9] Cree, "http://www.cree.com/power," [Online]. [Accessed 1411 2012].

[10] R. Shillington, P. Gaynor, M. Harrison and B. Heffernan, "Applications of silicon carbide JFETs in power converters," Universities Power Engineering Conference (AUPEC), 2010 20th Australasian, pp. 1-6, Dec. 2010.

[11] R. Mousa, D. Planson, H. Morel, B. Allard and C. Raynaud, "Modeling and high temperature characterization of SiC-JFET," Power Electronics Specialists Conference, 2008. PESC 2008. IEEE, pp. 3111-3117, 2008.

[12] T. Friedli, S. Round, D. Hassler and J. Kolar, "Design and Performance of a 200-kHz All-SiC JFET Current DC-Link Back-to-Back Converter," Industry Applications, IEEE Transactions on, vol.
45, no. 5, pp. 1868-1878, Sept. 2009.

[13] D. Aggeler, F. Canales, J. Biela and J. Kolar, "Dv/Dt-Power Control Methods for the SiC JFET/Si MOSFET Cascode," Power Electronics, IEEE Transactions on, vol. 28, no. 8, pp. 4074-4082, Aug. 2013.

[14] D. Peftitsis, R. Baburske, J. Rabkowski, J. Lutz, G. Tolstoy and H.-P. Nee, "Challenges Regarding Parallel Connection of SiC JFETs," Power Electronics, IEEE Transactions on, vol. 28, no. 3, pp. 1449-1463, , March 2013.

[15] B. Wrzecionko, D. Bortis, J. Biela and J. W. Kolar, "Novel AC-Coupled Gate Driver for Ultrafast Switching of Normally Off SiC JFETs," Power Electronics, IEEE Transactions on, vol. 27, no. 7, pp. 3452-3463, July 2012.

[16] Y. Durrani, E. Aeloiza, L. Palma and P. Enjeti, "An Integrated Silicon Carbide (SiC) Based Single Phase Rectifier with Power Factor Correction," Power Electronics Specialists Conference, 2005. PESC '05. IEEE 36th, pp. 2810-2816, 2005.

[17] F. Guedon, S. Singh, R. McMahon and F. Udrea, "Boost Converter With SiC JFETs: Comparison With CoolMOS and Tests at Elevated Case Temperature," Power Electronics, IEEE Transactions on, vol. 28, no. 4, pp. 1938-1945, April 2013.

[18] L. Huber, Y. Jang and M. Jovanovic, "Performance Evaluation of Bridgeless PFC Boost Rectifiers," Power Electronics, IEEE Transactions on, vol. 23, no. 3, pp. 1381-1390, May 2008.

[19] H.-Y. Tsai, T.-H. Hsia and D. Chen, "A Family of Zero-Voltage-Transition Bridgeless Power-FactorCorrection Circuits With a Zero-Current-Switching Auxiliary Switch," Industrial Electronics, IEEE Transactions on, vol. 58, no. 5, pp. 1848-1855, May 2010.

[20] Y. Jang and M. Jovanovic, "A Bridgeless PFC Boost Rectifier With Optimized Magnetic Utilization," Power Electronics, IEEE Transactions on, vol. 24, no. 1, pp. 85-93, Jan. 2009.

[21] K. Mino, H. Matsumoto, S. Fujita, Y. Nemoto, D. Kawasaki, R. Yamada and N. Tawada, "Novel bridgeless PFC converters with low inrush current stress and high efficiency," Power Electronics Conference (IPEC), 2010 International, pp. 17331739, Jun. 2010.

[22] Y. H. a. M. Mino, "High-density bidirectional rectifier for next generation 380-V DC distribution system," Applied Power Electronics Conference and Exposition (APEC), 2012 Twenty-Seventh Annual IEEE , pp. 2455-2460, Feb. 2012.

[23] C. Cass, R. Burgos, F. Wang and D. Boroyevich, "Three-phase AC Buck Rectifier using Normally- 
On SiC JFETs at $150 \mathrm{kHz}$ Swtiching Frequency," Power Electronics Specialists Conference, 2007. PESC 2007. IEEE, pp. 2162-2167, 2007.

[24] L. Huber and M. Jovanovic, "Performance evaluation of synchronous rectification in front-end full-bridge rectifiers," Applied Power Electronics Conference and Exposition (APEC), 2012 TwentySeventh Annual IEEE, pp. 310-316, Feb-2012.

[25] W. Zhong, S. Hui, W. Ho and X. Liu, "Using SelfDriven AC-DC Synchronous Rectifier as a Direct Replacement for Traditional Power Diode Rectifier," Industrial Electronics, IEEE Transactions on, vol. 59, no. 1, pp. 392-401, Jan. 2012.

[26] J. J. Zhang and IR, "GaN-based device cascoded with an integrated FET/Schottky diode device". USA Patent US8084783B2, 27 Dec 2011. 